\title{
Test of Accuracy of the Generalized Boundary Conditions in the Scattering by Thin Dielectric Strips
}

\author{
Alexander I. Nosich, ${ }^{1}$ Olga V. Shapoval, ${ }^{1}$ Ilya O. Sukharevsky, ${ }^{2}$ Ayhan Altintas ${ }^{2}$ \\ ${ }^{1}$ Laboratory of Micro and Nano-Optics, Institute of Radio-Physics and Electronics NASU, Kharkiv 61085, Ukraine \\ Email: anosich@yahoo.com \\ ${ }^{2}$ Department of Electrical and Electronics Engineering, Bilkent University, 06800 Ankara, Turkey
}

\begin{abstract}
The two-dimensional (2D) scattering of the $\mathrm{E}$ and $\mathrm{H}$ polarized plane electromagnetic waves by a free-standing thinner than the wavelength dielectric strip is considered numerically. Two methods are compared: singular integral equations (SIE) on the strip median line obtained from the generalized boundary conditions for a thin dielectric layer and Muller boundary integral equations (BIE) for arbitrarily thick strip. The comparison shows the domain of acceptable accuracy of approximate model derived for thin dielectric strips.
\end{abstract}

Keywords - thin strip, generalized boundary conditions, scattering, integral equations.

\section{INTRODUCTION}

Both natural and man-made flat thin dielectric scatterers are frequently met in the electromagnetic-wave technologies and applications across wide spectrum of frequencies from microwaves to optics. Among them there are tree leaves, screens and panels, dielectric-patch antennas, and high-Q whispering-gallery-mode resonators, to name only a few. In fact, such objects are quite common building-blocks in various electromagnetic systems because they possess distinctive resonant properties and are relatively easy manufactured with today's printing and lithography techniques. One of the simplest scatterers of this type is a flat dielectric strip (Fig. 1).

Prediction of the electromagnetic behavior of a thin dielectric strip is more difficult task than it can appear. In part, this is because the well-known computational techniques developed for perfectly electrically conducting (PEC) strips are not applicable. The other difficulty arises from the combination of a comparable or larger than the wavelength width and a fraction-of-wavelength thickness. This makes a burden for any numerical code based on BIE. A volume integral-equation (VIE) method may seem free of the contour discretization problems and hence more attractive [1]. However a closer inspection shows that an electric-field VIE leads to a stable and convergent algorithm only in the Epolarization case because this VIE has a Hankel function in the kernel and hence is a Fredholm second-kind equation. In the H-polarization case analogous VIE is a hyper-singular equation, and therefore a denser meshing of the strip crosssection does not provide convergence.

Hence it is not a surprise that since the 1980s the scattering by thin material scatterers has been treated using an empiric albeit well-understood approach. It is based on the shrinking

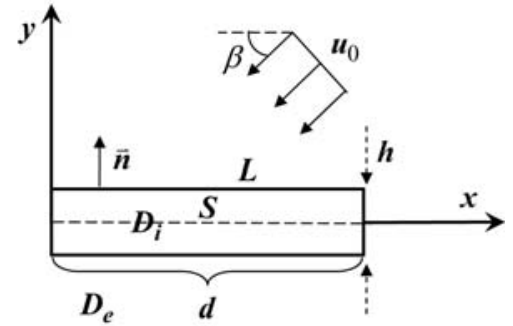

Fig. 1. Cross-sectional view of a free-standing thin dielectric strip of relative permittivity $\varepsilon$, width $d$ and thickness $h$, illuminated by a plane wave incident under the angle $\beta$.

of the scatterer to its median line or plane, introduction of effective electric and magnetic currents, and use of the generalized boundary condition (GBC), which somehow takes account of the actual thickness of scatterer.

The GBC was first published in [1] and then considered in many other papers - see [2-8]. It has been applied to study the scattering by thin half-planes and strips in [9-17]. In the early works, it had been established that the effective electric and magnetic currents were decoupled and satisfied independent IEs. Their solutions were normally obtained using the moment method (MM) with local basis functions although in [10] a Wiener-Hopf technique was applied for a wide strip.

However, one of these two IEs is a hyper-singular equation and therefore the convergence of MM solution is absent. This does not mean that this SIE cannot be solved numerically simply it should be solved in different manner. For instance, a meshless Nystrom-type discretization that provides guaranteed convergence and small size matrices can be used.

However, since the GBC is derived for an infinite thin material slab, its applicability for shorter and thicker finite strips, near the resonances, and at the grazing (edge-on) incidence can be questioned. To establish the domain of validity of the GBC-SIE approach one should compare its numerical results with the reference ones, i.e. the data obtained with some independent method. To be trusted, such method must not assume the strip to be thin and must have mathematically guaranteed convergence when taking larger the discretization order.

The choice of the reference code is therefore conditioned by the demand of guaranteed convergence. As mentioned, the VIE method has convergence only in E-case and therefore is not a perfect candidate. The BIE techniques deal with more 
economic discretizations than VIE. They can serve as a source of reference data if, besides of guaranteed convergence, they are free from the spurious real-valued eigenvalues [18]. This is true for the Muller BIE (MBIE) that is actually a pair of coupled Fredholm second-kind IEs [19-22].

Therefore we use MBIE to validate GBC-SIE. Here, the Nystrom-type meshless discretization using interpolation polynomials and quadrature formulas is known as efficient solution technique [20,21]. We have adapted it for a piecewise smooth contour of our strip scatterer.

\section{Problem Statement AND GBC-SIE MethoD}

Assume that electromagnetic plane wave is incident at the angle $\beta$ on a dielectric strip as shown in Fig. 1. Denote the $z$ component of the $\mathrm{E}$ or $\mathrm{H}$ field as $U^{i n c}(\vec{r})=e^{-i k_{0}(x \cos \beta+y \sin \beta)}$, where $\vec{r}=(x, y), k_{0}=\omega / c$, and $c$ is the free-space light velocity. The strip is characterized with the width $d$, thickness $h$. It has rectangular cross-sectional contour $L$ and inner domain $D$ filled with material having complex relative permittivity $\varepsilon$. The host medium is free space.

In the presence of strip, the total field $U(\vec{r})$ is a sum of $U^{i n c}(\vec{r})$ and the scattered field, $U^{s c}(\vec{r})$. The latter function must satisfy the boundary-value problem (BVP), which involves Helmholtz equations inside and outside the strip and the continuity of tangential components across $L$. For uniqueness, $U^{s c}(\vec{r})$ must also satisfy Sommerfeld radiation condition and condition of local energy finiteness.

The GBC-SIE method does not follow from original BVP in mathematically justified manner. Instead it uses certain physical assumptions for thin strips and hence is empirical. Namely, it is assumed that $k_{0} h<<1$ and strip's cross-section $D$ can be shrunk to the median line, $S=\{0 \leq x \leq d, y=0\}$, the field inside the strip can be excluded from consideration, and the following GBC can be imposed on $S$ (see [6] for details):

$$
\begin{gathered}
(1 / 2)\left[\vec{E}_{T}^{+}(x, 0)+\vec{E}_{T}^{-}(x, 0)\right]=R Z_{0} \vec{n} \times\left[\vec{H}_{T}^{+}(x, 0)-\vec{H}_{T}^{-}(x, 0)\right], \\
(1 / 2)\left[\vec{H}_{T}^{+}(x, 0)+\vec{H}_{T}^{-}(x, 0)\right]=-Q Z_{0}^{-1} \vec{n} \times\left[\vec{E}_{T}^{+}(x, 0)-\vec{E}_{T}^{-}(x, 0)\right],
\end{gathered}
$$

This pair of equations was originally derived for an infinite thin material slab illuminated by a plane wave [6]. Here, $\vec{n}=(0,1)$ is the unit normal vector, $Z_{0}$ is the free-space impedance, and the indices \pm correspond to the field limit values at the top and bottom sides of the strip, respectively. Equations (1) are the Ohm's law for the effective surface currents. Therefore the coefficients $R Z_{0}$ and $Q Z_{0}^{-1}$ are called the electric and magnetic resistivities. They have several forms. If the contrast is high, $|\varepsilon|>>1$, then [2,5-8],

$$
R=\frac{i}{2 \varepsilon^{1 / 2}} \cot \left(\frac{k_{0} h \varepsilon^{1 / 2}}{2}\right), Q=\frac{i \varepsilon^{1 / 2}}{2} \cot \left(\frac{k_{0} h \varepsilon^{1 / 2}}{2}\right)
$$

If, alternatively, the contrast is low, $k_{0} h|\varepsilon-1| \ll 1$, then [9]

$$
R=i\left[\varepsilon^{1 / 2}(\varepsilon-1) k_{0} h\right]^{-1}, \quad Q=i \varepsilon^{1 / 2}\left[(\varepsilon-1) k_{0} h\right]^{-1}
$$

A modified version of (2) has been proposed in [8]. It takes into account the phase compensation for the reduction of the slab area to the median line of zero thickness,

$$
R^{*}=\frac{\vartheta-R-\vartheta^{2} R}{4 \vartheta R-\vartheta^{2}-1}, \quad Q^{*}=\frac{\vartheta-Q-\vartheta^{2} Q}{4 \vartheta Q-\vartheta^{2}-1}
$$

where $\vartheta=i \cot \left(k_{0} h / 4\right)$, and $R$ and $Q$ are the same as in (2).

When GBC (1) is imposed at a finite strip, it must be supplemented with the condition of local finiteness of power and the radiation condition. The field singularities at the edges of a resistive strip have been analyzed in [23]; they are different from the right-angle dielectric wedge.

The scattered field is sought for as a sum of single-layer and double-layer potentials,

$$
U^{s c}(\vec{r})=k_{0} \int_{S} v\left(\vec{r}^{\prime}\right) G\left(\vec{r}, \vec{r}^{\prime}\right) d \vec{r}^{\prime}+\int_{S} w\left(\vec{r}^{\prime}\right) \frac{\partial G\left(\vec{r}, \vec{r}^{\prime}\right)}{\partial \vec{r}^{\prime}} d \vec{r}^{\prime},
$$

where $U(\vec{r})$ is either $H_{z}(\vec{r})$ or $E_{z}(\vec{r})$ depending on the polarization, and $G\left(\vec{r}, \vec{r}^{\prime}\right)$ is the free-space Green's function. From the properties of the limit values of potentials it follows that unknown functions are $v(\vec{r})=\partial / \partial \vec{n}(\vec{r})\left[U^{+}(\vec{r})-U^{-}(\vec{r})\right]$ and $w(\vec{r})=U^{+}(\vec{r})-U^{-}(\vec{r})$.

Using (5) in GBC (1) yields two independent IEs of the second kind with the same integration domain. After using the strip parameterization as $x=d(t+1) / 2, y=0$ with $t \in[-1,1]$ and new unknown function $\tilde{w}(t)=w(t)\left(1-t^{2}\right)^{-1 / 2}$, we obtain in the H-polarization case,

$$
\begin{gathered}
4 Q v\left(t_{0}\right)+\kappa \int_{-1}^{1} v(t) H_{0}^{(1)}\left(\kappa\left|t-t_{0}\right|\right) d t=f_{v}\left(\kappa, t_{0}\right) \\
4 R \tilde{w}\left(t_{0}\right) \sqrt{1-t_{0}^{2}}+\int_{-1}^{1} \tilde{w}(t) \sqrt{1-t^{2}} \frac{H_{1}^{(1)}\left(\kappa\left|t-t_{0}\right|\right)}{\left|t-t_{0}\right|} d t=f_{w}\left(\kappa, t_{0}\right)
\end{gathered}
$$

In the case of the E-polarized wave, the quantities $R$ and $Q$ exchange places. Here, $\kappa=k_{0} d / 2$ and the right-hand-part terms $f_{v}(\kappa, t)=4 i e^{-i \kappa(t+1) \cos \beta}$ and $f_{w}(\kappa, t)=4 \sin \beta e^{-i \kappa(t+1) \cos \beta}$ are defined by the incident wave.

Further we discretize SIEs (6) and (7) using a Nystrom-type method, i.e. approximating the unknowns by polynomials and using two quadrature rules of interpolation type of the order $N$ [13]. The theorems on quadratures ensure convergence of such numerical scheme with the rate of at least $1 / N$ if $N \rightarrow \infty$. This makes the resulting algorithm very economic and enables one to study not only the scattering by single strips but also by the finite and infinite gratings of them [14-17]. All data presented below have been computed with $10^{-4}$ accuracy that was achieved with $N=50$. 


\section{MulLer BIE MethoD}

MBIE are derived using the algebraic manipulations with Green's formulas and theorems of the potential theory [19]. In the 2D case, such derivation can be found in $[20,21]$ and the resulting equations have the following form:

$$
\begin{gathered}
U(\vec{r})+\int_{L} K_{11}\left(\vec{r}, \vec{r}^{\prime}\right) U\left(\vec{r}^{\prime}\right) d \vec{r}^{\prime}-\int_{L} K_{12}\left(\vec{r}, \vec{r}^{\prime}\right) V\left(\vec{r}^{\prime}\right) d \vec{r}^{\prime}=U^{\text {inc }}(\vec{r}), \\
\frac{1+p}{2} V(\vec{r})+\int_{L} K_{21}\left(\vec{r}, \vec{r}^{\prime}\right) U\left(\vec{r}^{\prime}\right) d \vec{r}^{\prime}-\int_{L} K_{22} V\left(\vec{r}^{\prime}\right) d \vec{r}^{\prime}=V^{\text {inc }}(\vec{r}), \vec{r} \in L
\end{gathered}
$$

Here, $V(\vec{r})=\partial U(\vec{r}) / \partial n$ is the limit value of the normal derivative of the total field function on the closed contour $L$ of the scatterer from the inner side of it, the normal unit vector $\vec{n}$ is directed to the outer domain (free space), $d \vec{r}^{\prime}$ is the elementary arc length, and the constant $p$ is 1 in the Epolarization case and $1 / \varepsilon$ in the H-polarization case.

The kernel functions of MBIE are given by the linear combinations of the Green's functions of the homogeneous media with the wavenumbers $k_{e}=k_{0}$ and $k_{i}=k_{0} \sqrt{\varepsilon}$, and of their first-order and second-order normal derivatives [19-22].

As known [20,21], if $L$ is a Lyapunov curve then the kernel functions have only logarithmic singularities, and (8), (9) together are a Fredholm second kind IE. This guarantees convergence of conventional MM schemes of its solution. However, if $L$ is a piece-wise Lyapunov curve, then some additional steps are needed to build a convergent code.

We subdivide the strip contour $L$ into four straight intervals, extract logarithmic singularities from the kernels, and apply quadratures on each smooth segment. The discretization performed in this manner is not sensitive to the irregularities of the contour provided that the interpolation nodes do not coincide with edge points. After introducing new continuous kernels (with the extracted singularities) we divide the segments of $L$ into $N$ sub-sections, and assume that unknown functions are constants at each sub-section. Numerical integration based on the rectangle rule leads to a matrix equation with respect to the unknowns. To reach the accuracy of the order of $10^{-4}$ in the far field, we have taken, in computations, $N=600$. Verification of solution has been performed by comparing the results with [1].

\section{Numerical Results}

For systematic comparisons, we have selected the radar scattering cross-section (RCS) and the total scattering crosssection (TSCS). Note that these quantities are computed from the far-field data [13]. They are not sensitive to the field behavior at the strip edges because the condition of local power finiteness tells that "the edges do not radiate." We have considered the cases of two alternative polarizations.

\section{- E-polarization}

First of all, to validate the results of MBIE, we have compared them with those obtained by a different method.

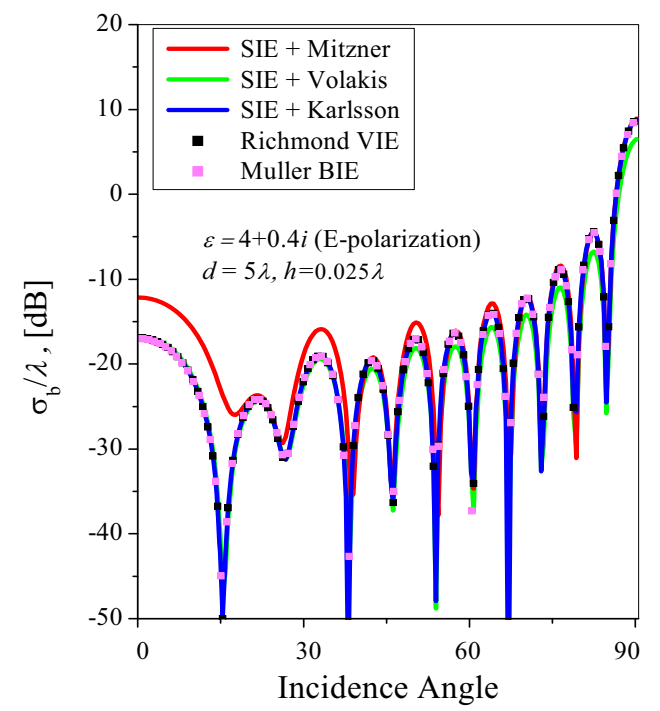

Fig. 2. Comparison with Richmond VIE solution [1]. Normalized RCS as a function of the incidence angle of the E-wave for the scattering by a strip with $d=5 \lambda, h=0.025 \lambda$ (i.e. $h / d=0.005$ ) and $\varepsilon=4.0+0.4 i$.

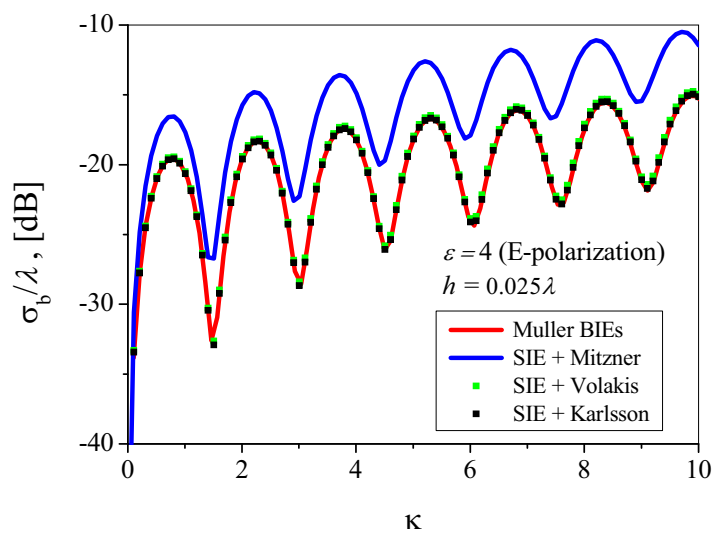

Fig. 3. Normalized RCS versus the normalized frequency $\kappa$ for the edge-on scattering of E-wave $(\beta=0)$ by a dielectric strip of $h=0.025 \lambda$ with $\varepsilon=4.0$. The Muller BIE curve graphically overlaps with Richmond's VIE data [1].

Such reference data are available in the E-case only, as a numerical solution of the VIE obtained by Richmond [1]. In Fig. 2, we show the dependences of TSCS for a lossy strip with $d=2 \lambda, \varepsilon=4(1-0.1 i)$, and thickness $h / d=0.005$ on the incidence angle. As one can see, the MBIE and VIE curves overlap for all angles including the least favorable case of the edge-on illumination $(\beta=0)$. Other three curves correspond to the three versions of GBC-SIE results computed using equations (2), (3) and (4) for the resistivities.

The case of the edge-on incidence is studied in more details in Fig. 3 where the frequency scans of TCSC are presented. As visible, all three models correctly predict periodic resonances in RCS caused by the interference between the reflections from the leading and the trailing edges of strip. The notcompensated model of (2) is the least accurate showing a $5 \mathrm{~dB}$ to $10 \mathrm{~dB}$ error in this range.

Dependences of TSCS on the relative thickness of strip are shown in Fig. 4. One can see that the error in Karlsson's model does not exceed $1 \%$ for $h / d<0.1$, in contrast to $10 \%$ 
for the model of (2). The low-contrast model of (3) is good only for very thin strips: it can be used with a $2 \%$ or better accuracy if $\kappa<2.5$, i.e. where $k_{0} h|\varepsilon-1|<0.5$.

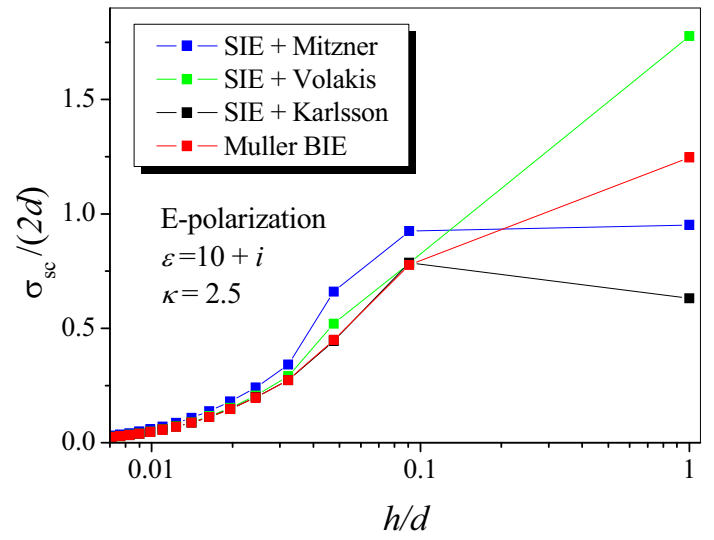

Fig. 4. Normalized TSCS versus $d / h$ for a lossy dielectric strip with of $\varepsilon=10$ $+i$ for $\kappa=2.5$ under the normally incident $(\beta=\pi / 2)$ E-polarized wave.

\section{- H-polarization}

In Fig. 5, we show the dependences of TSCS on the relative thickness, $h / d$ for the H-case scattering. The compensated high-contrast model of (4) agrees very well with the MBIE reference data. If $h / d<0.1$, the models of (4) and (3) provide $4 \%$ and $10 \%$ accuracy, respectively.

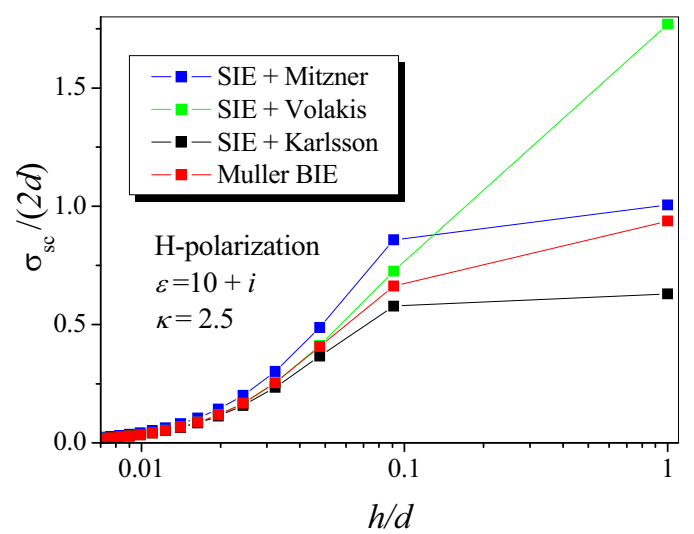

Fig. 5. The same as in Fig. 6 however for the H-polarization case.

\section{CONCLUSIONS}

Two numerical approaches to the analysis of the 2D scattering by thin dielectric strips have been compared. Approximate empiric method based on the GBC and the median-line SIE with a Nystrom-type discretization has been validated via comparison with accurate method based on the Muller BIE discretized with another Nystrom method. In either of two polarizations, the GBC-SIE with compensated high-contrast model of [8] for the resistivity coefficients is able to provide the values of TSCS with accuracy within $4 \%$ both off and in the resonances provided that $h / d<0.05$.

\section{REFERENCES}

[1] J. H. Richmond, "Scattering by a thin dielectric strip," IEEE Trans. Antennas Propag., vol. AP-33, no 1, pp. 64-68, 1985.

[2] K. M. Mitzner, "Effective boundary conditions for reflection and transmission by an absorbing shell of arbitrary shape," IEEE Trans. Antennas Propag., vol. 16, pp. 706-712, 1968.

[3] R. F. Harrington and J. R. Mautz, "An impedance sheet approximation for thin dielectric shells," IEEE Trans. Antennas Propag., vol. 36, pp. 531-534, 1975 (in fact, this paper deals with the resistive sheet).

[4] T. B. A. Senior and J. L. Volakis, "Sheet simulation of a thin dielectric layer," Radio Sci., vol. 22, no. 7, pp. 1261-1272, 1987.

[5] G. Bouchitté, "Analyse limite de la diffraction d'ondes électromagnétiques par une structure mince", C.R. Acad. Paris, t. 311, pp. 51-56, 1990.

[6] E. Bleszynski, M. Bleszynski, and T. Jaroszewicz, "Surface-integral equations for electromagnetic scattering from impenetrable and penetrable sheets," IEEE Antennas Propag. Mag., vol. 36, pp. 14-25, 1993.

[7] Z. Nazarchuk and K. Kobayashi, "Mathematical modelling of electromagnetic scattering from a thin penetrable target," Progress Electromag. Res., vol. 55, pp. 95-116, 2005.

[8] A. Karlsson, "Approximate boundary conditions for thin structures," IEEE Trans. Antennas Propag., vol. 57, no 1, pp. 144-148, 2009.

[9] J. L. Volakis, "High-frequency scattering by a thin material half-plane and strip," Radio Science, vol. 23, no 3, pp. 450-462, 1988.

[10] S. Dowerah and A. Chakrabarti, "Extinction cross-section of a dielectric strip," IEEE Trans. Antennas Propag., vol. 36, no 5, pp. 696-706, 1988.

[11] T. L. Zinenko, A. I. Nosich, and Y. Okuno, "Plane wave scattering and absorption by resistive-strip and dielectric-strip periodic gratings," IEEE Trans. Antennas Propagat., vol. 46, pp. 1498-1505, 1998.

[12] T.L. Zinenko and A.I. Nosich, "Plane wave scattering and absorption by flat gratings of impedance strips," IEEE Trans. Antennas Propagat., vol. 54, no 7, pp. 2088-2095, 2006

[13] O. V. Shapoval, R. Sauleau, and A. I. Nosich, "Scattering and absorption of waves by flat material strips analyzed using generalized boundary conditions and Nystrom-type algorithm," IEEE Trans. Antennas Propag., vol. 59, no 9, pp. 3339-3346, 2011.

[14] T.L. Zinenko, M. Marciniak, and A.I. Nosich, "Accurate analysis of light scattering and absorption by an infinite flat grating of thin silver nanostrips in free space using the method of analytical regularization," IEEE J. Sel. Top. Quant. Electron., vol. 19, no 3, art. 9000108/8, 2013.

[15] O.V. Shapoval and A.I. Nosich, "Finite gratings of many thin silver nanostrips: optical resonances and role of periodicity," AIP Advances, vol. 3, no 4, pp. 042120/13, 2013.

[16] O. V. Shapoval, R. Sauleau, and A. I. Nosich, "Modeling of plasmon resonances of multiple flat noble-metal nanostrips with a median-line integral equation technique," IEEE Trans. Nanotechnology, vol. 12, no 3, pp. 442-449, 2013.

[17] O.V. Shapoval, J. Ctyroky, and A.I. Nosich, "Resonance effects in the optical antennas shaped as finite comb-like gratings of noble-metal nanostrips," Proc. SPIE, vol. 8781, pp. 87810U/8, 2013.

[18] A. F. Peterson, "The 'interior resonance' problem associated with surface integral equations of electromagnetics: numerical consequences and a survey of remedies," Electromagnetics, vol. 10, pp. 293-312, 1990.

[19] C. Muller, Foundations of the Mathematical Theory of Electromagnetic Waves, Berlin, Springer, 1969 (German edition, 1957).

[20] S.V. Boriskina, T.M. Benson, P. Sewell, and A.I. Nosich, "Q factor and emission pattern control of the WG modes in notched microdisk resonators," IEEE J. Sel. Top. Quant. Electron., vol. 12, pp. 52-58, 2006.

[21] E. I. Smotrova, V. Tsvirkun, I. Gozhyk, C. Lafargue, C. Ulysse, M. Lebental, and A. I. Nosich, "Spectra, thresholds and modal fields of a kite-shaped microcavity laser," J. Optical Society America B, vol. 40, no 6, pp. 1732-1742, 2013.

[22] J. L. Tsalamengas, "Exponentially converging Nystrom method in oblique diffraction of arbitrarily polarized waves by bianisotropic/chiral cylinders with arbitrary smooth cross section," IEEE Trans. Antennas Propag., vol. 61, no 7, pp. 3362-3673, 2013.

[23] I. M. Braver, P. S. Fridberg, K. L. Garb, and I. M. Yakover, "The behavior of the electromagnetic field near the edge of a resistive halfplane," IEEE Trans. Antennas Propag., vol. 36, pp. 1760-1768, 1988. 